\title{
Neurocognitive functioning in community youth with suicidal ideation: gender and pubertal effects
}

\author{
Ran Barzilay, Monica E. Calkins, Tyler M. Moore, Rhonda C. Boyd, Jason D. Jones, Tami D. Benton,
} Maria A. Oquendo, Ruben C. Gur and Raquel E. Gur

\section{Background}

Although there are extensive data on clinical psychopathology in youth with suicidal ideation, data are lacking regarding their neurocognitive function.

\section{Aims}

To characterise the cognitive profile of youth with suicidal ideation in a community sample and evaluate gender differences and pubertal status effects.

\section{Method}

Participants ( $N=6151$, age 11-21 years, 54.9\% females) from the Philadelphia Neurodevelopmental Cohort, a non-help-seeking community sample, underwent detailed clinical evaluation. Cognitive phenotyping included executive functioning, episodic memory, complex reasoning and social cognitive functioning. We compared participants with suicidal ideation $(N=672)$ and without suicidal ideation $(N=5479)$. Regression models were employed to evaluate differences in cognitive performance and functional level, with gender and pubertal status as independent variables. Models controlled for lifetime depression or general psychopathology, and for covariates including age and socioeconomic status.

\section{Results}

Youth with suicidal ideation showed greater psychopathology, poorer level of function but better overall neurocognitive performance. Greater functional impairment was observed in females with suicidal ideation (suicidal ideation $\times$ gender interaction, $t=3.091, P=0.002$ ). Greater neurocognition was associated with suicidal ideation post-puberty (suicidal ideation $\times$ puberty interaction, $t=3.057, P=0.002$ ). Exploratory analyses of specific neurocognitive domains showed that suicidal ideation-associated cognitive superiority was more prominent in post-pubertal males compared with females (Cohen's $d=0.32$ and $d=0.11$, respectively) across all cognitive domains.

\section{Conclusions}

Suicidal ideation was associated with poorer functioning yet better cognitive performance, especially in post-pubertal males, as measured by a comprehensive cognitive battery. Findings point to gender and pubertal-status specificity in the relationship between suicidal ideation, cognition and function in youth.

\section{Declaration of interest}

R.B. serves on the scientific board and reports stock ownership in 'Taliaz Health', with no conflict of interest relevant to this work. M.A.O. receives royalties for the commercial use of the Columbia-Suicide Severity Rating Scale from the Research Foundation for Mental Hygiene. Her family owns stock in Bristol-Myers Squibb. All other authors declare no potential conflict of interest.

\section{Keywords}

Suicidal ideation; cognition; gender differences; developmental psychopathology.

\section{Copyright and usage}

(c) The Royal College of Psychiatrists 2019.
Youth suicidal behaviour is a major health concern, ${ }^{1}$ as suicide is the second leading cause of death in youth across the globe. ${ }^{2}$ Suicidal behaviour in youth is considered a spectrum spanning thoughts about death or dying, suicidal ideation, attempt planning and suicide attempt, which may result in death. ${ }^{3}$ Previous studies in community youth have suggested that a significant proportion of youths with suicidal ideation will proceed to a suicide attempt ${ }^{4-6}$ and that childhood depression is a significant mediator of risk. ${ }^{7}$ Others suggest that suicidal ideation is an independent indicator for longstanding mental health risk, even after controlling for adolescent depression, implicating unique mechanisms that may underpin suicidal ideation itself. ${ }^{8}$ These findings highlight the need to better understand brain and behaviour measures that are associated with suicidal ideation in youth. Whereas association of suicidal ideation with greater psychopathology and poorer functional outcome is well established in youth, ${ }^{6,7,9}$ literature is inconclusive regarding how suicidal ideation in youth is related to cognitive performance, e.g. as obtained from standard neuropsychological testing. To date, some studies described poorer cognitive function associated with suicidal ideation, ${ }^{10}$ but recent data suggest otherwise. ${ }^{11}$ Whereas youth suicidal ideation is associated with established gender and puberty effects, ${ }^{4,5}$ limited data exist on the effects of gender and pubertal status in associations between suicidal ideation and neurocognitive function. ${ }^{12}$ Investigation of neurocognitive functioning in youth with suicidal ideation may reveal distinct neurocognitive phenotypes that are uniquely associated with suicidal ideation and may help identify suicide-related risk. Furthermore, some neurocognitive phenotypes might be considered as targets of early or preventive interventions. The Philadelphia Neurodevelopmental Cohort (PNC) is a large community sample, well balanced for males/females and pre-/post-pubertal status, with deep phenotyping that includes suicidal ideation and detailed neurocognitive evaluation in youth not seeking mental-health help. Our aim was to evaluate neurocognitive function in youth with suicidal ideation across multiple domains in relation to gender and puberty, and to test whether the suicidal ideation-neurocognitive function association is independent of the effects of depression or other general psychopathology.

\section{Method}

\section{Participants}

The PNC is a collaboration between the Children's Hospital of Philadelphia and the Brain Behavior Laboratory at the University of Pennsylvania. ${ }^{13}$ Enrolment criteria included: (a) age 8-21 years, (b) ambulatory in stable health, (c) proficient in English, (d) physically and cognitively capable of completing study procedures, and (e) absence of a significant physical condition or developmental delay that impairs motility or cognition (e.g. paresis or palsy, intellectual disability). Participants were recruited from a pool $(N=50293)$ 
of children previously genotyped as part of a genomic study at the Children's Hospital of Philadelphia healthcare network, which extends to $>30$ clinical community sites in the tri-state area of Pennsylvania, New Jersey and Delaware, USA. Participants were not recruited from psychiatric clinics and the sample is not enriched for those seeking mental health services. Based on review of electronic medical records or follow-up phone contact, potential participants from this pool were excluded if they were not proficient in English, had significant developmental delays or other conditions that would interfere with their ability to complete study procedures or if they could not be contacted. From the remaining pool, 13598 individuals were invited, 2699 declined (18.9\%), 1401 were excluded and 9498 youths (age 8-21) were enrolled. The large community sample is racially $(56 \%$ White, $33 \%$ African American and $11 \%$ other) and socioeconomically diverse, ${ }^{14}$ reflecting the regional demographics of the greater Philadelphia area. The clinical assessment was administered to collateral informants who were caregivers or legal guardians for participants aged 8-10, to both participant and collaterals for participants aged 11-17 and solely to participants aged 18-21. Study participants were compensated for their time including travel. For the current analyses we included only participants' interviews (and not those of collaterals) and therefore only data from participants aged $11-21$ were analysed $(N=7054)$ due to low agreement between youth and parent report of suicidal ideation in the PNC. ${ }^{15}$ For 108 participants (1.53\% of sample), interview sections including suicidal ideation items were missing and therefore they were excluded from analyses.

\section{Ethics statement}

This study was performed in accordance with the Declaration of Helsinki. After complete description of the study, written informed consent was obtained from participants aged $\geq 18$, and written assent and parental permission were obtained from children aged $<18$ and their parents/legal guardian. The study was approved by the University of Pennsylvania and the Children's Hospital of Philadelphia Institutional Review Boards (protocol number 810336).

\section{Clinical evaluation}

Psychopathology symptoms were evaluated by trained and supervised assessors, using a structured screening interview (GOASSESS), as detailed elsewhere, ${ }^{13}$ which was based on the Kiddie Schedule for Affective Disorders and Schizophrenia (K-SADS). ${ }^{16}$ Lifetime depressive episode diagnoses were determined if symptoms were endorsed with frequency and duration meeting DSM-IV (1994) episode criteria, accompanied by significant distress or impairment.

Lifetime suicidal ideation was assessed with a single question in which the participant was asked, 'Have you ever thought a lot about killing yourself?' Immediately before that question, participants were also asked in a separate question whether they had ever 'thought a lot about death or dying'. Participants who endorsed either suicidal ideation or thoughts of death/dying were asked if the thoughts were current (within the past month) and, if so, a clinician was contacted before the end of the visit for further follow-up.

Although literature supports the consideration of thoughts about death as part of the suicidal behaviour spectrum, ${ }^{3}$ to avoid misclassification of suicidal ideation and the control group, we excluded from the main analyses the participants who endorsed having ever 'thought a lot about death or dying' but did not endorse suicidal ideation $(N=795,11.3 \%$ of study sample). Sensitivity analyses that included participants who endorsed death/dying but denied suicidal ideation, and compared youths who endorsed any of the two items (thoughts about suicide or thoughts of death/dying) with controls (youths who denied both items), yielded similar findings and results are presented in the Supplementary Material available at https://doi.org/10.1192/bjp.2019.55. The decision to exclude the participants who endorsed thinking of death/dying but did not endorse suicidal ideation from the main analysis was based on clinical observations that some youth endorse thinking of death or dying due to thoughts that are not related to the suicide spectrum (e.g. death of a relative, fears associated with a medical illness or other lifethreatening event they or someone close to them had experienced).

To generate psychopathology factor scores, we used item-wise (i.e. symptom-level) psychopathology responses from GOASSESS across all assessed psychopathology domains. An exploratory factor analysis was conducted, extracting four factors (using 110 items), as previously described. ${ }^{17}$ The items used to calculate the factor scores did not include the suicidal ideation item (independent variable in the current analysis) or the item regarding ever thought a lot about death/dying. This exploratory factor analysis was then used to assign items to factors for a confirmatory factor analysis. The confirmatory factor analysis was estimated using a Bayesian estimator in Mplus, version 7.1 (for Windows). As predicted by theory and supported by initial exploratory models, the four factors primarily represent anxious-misery (mood-anxiety) symptoms, psychosis spectrum symptoms, externalising behaviour symptoms (conduct and attention-deficit hyperactivity disorder) and fear symptoms (phobias). Factor scores were generated from these four confirmatory correlated-traits factors. Additionally, a bifactor model estimated a general psychopathology factor, representing the overall burden of psychopathology while controlling for the presence of specific symptom dimensions. ${ }^{17}$

Level of function was evaluated by the Children's Global Assessment Scale (C-GAS) ${ }^{18}$ and by the Occupational Functioning Scale (N6) of the Scale of Prodromal Symptoms (SOPS). ${ }^{19}$ A score greater than zero in the SOPS N6 item represents difficulties in age-appropriate role functions, such as school performance, and/or difficulties in relationships. Pubertal status was determined based on a computerised and privately administered self-report of genital development (Tanner score of five was considered post-pubertal). ${ }^{20}$

\section{Neurocognitive evaluation}

The 1 hour Penn Computerized Neurocognitive Battery (CNB) includes 14 tests assessing 5 neurobehavioural domains: (a) executive function: Penn Conditional Exclusion Test, Letter N-Back (NBACK) and Continuous Performance Test (CPT); (b) episodic memory (verbal, face and spatial memory tests); (c) complex cognition (language reasoning, nonverbal reasoning (Penn Matrix Reasoning Test, PMAT) and spatial processing (Penn Line Orientation Test, PLOT); (d) social cognition (emotion identification, emotion intensity differentiation and age differentiation); and (e) sensorimotor speed (motor and sensorimotor). ${ }^{21}$ The reading subtest of the fourth edition of the Wide Range Achievement Test (WRAT4) ${ }^{22}$ was administered to determine participants' ability to complete the battery and provide an IQ estimate. Here, we examined accuracy as a measure of performance. False positive responses in the CPT served as a proxy measure for impulsivity. ${ }^{23}$

\section{Statistical analysis}

Univariate comparisons between participants endorsing suicidal ideation ('suicide ideators') and denying suicidal ideation ('non-suicidal') youth were conducted using two-tailed $t$-tests or $\chi^{2}$ tests, as appropriate. For multivariate analyses, we performed binary logistic regressions to investigate the association of gender, pubertal status and their interaction with suicidal ideation as the dependent variable, controlling for age (puberty regressed) and socioeconomic status as covariates.

To evaluate differences between suicide ideators and non-suicidal youth across different psychopathology domains we performed a mixed model repeated measures (MMRM) analysis, treating 
psychopathology factor domains (anxious-misery, psychosis, externalising and fear) as a within-subject variable, controlling for age, gender and socioeconomic status. The effects of interest were the suicide $\times$ domain interactions. When significant, these interactions show that differences in psychopathology level between suicide ideators and non-suicidal youth varies among psychopathology domains.

To evaluate the association of suicidal ideation with function level and with overall cognitive performance accuracy, we conducted linear regression models with C-GAS score or CNB accuracy $Z$-score as the dependent variable and with suicidal ideation, gender, puberty and their interactions as the independent variables, controlling for age (puberty regressed), socioeconomic status, lifetime history of depressive disorder or general psychopathology factor score. Separate models controlled for race (White, African American and other) and a measure of parents' separation/divorce status based on the clinical interview, instead of socioeconomic status, due to their high correlation.

The association of suicidal ideation with impaired functioning, measured by the SOPS occupational function severity scale, was examined by binary logistic regression with impaired functioning (rating $>0$ ) as a dependent variable and suicidal ideation as the independent variable, controlling for gender, socioeconomic status, puberty and age (puberty regressed) and the general psychopathology factor score.

In an exploratory analysis to compare accuracy in specific cognitive tests, we performed $t$-tests between suicide ideators and non-suicidal youth in pre- and post-pubertal males and females. False-discovery rate (FDR) was used to correct for multiple testing. A two-tailed $P$-value $<0.05$ was considered statistically significant in all analyses. Statistical analyses were performed using SPSS Statistics 24 for Windows (IBM, Armonk, New York, USA), except for the MMRM ('nlme' package in $\mathrm{R}^{24}$ ).

\section{Data availability}

The authors had full access to the PNC data that were used in the current analysis.

\section{Results}

\section{Suicidal ideation rates $\times$ gender and pubertal status}

Suicidal ideation was reported by 672 (10.9\% of study sample) participants. Suicide ideators were older, of lower socioeconomic status, had higher rates of lifetime depressive episodes and greater overall general psychopathology (Table 1). As expected, suicidal ideation was associated with post-pubertal status, with a gender $\times$ puberty interaction manifested by a higher rate of suicidal ideation in post-pubertal females compared with males (15.7 v. 12.4\%, respectively) that was not observed in pre-pubertal youth (between 7 and $8 \%$ males and females had suicidal ideation; Fig. 1).

\section{Association of psychopathology with suicidal ideation}

We investigated the association of suicidal ideation with psychopathology in four domains using factor scores of mood-anxiety, psychosis spectrum, externalising symptoms and fear. ${ }^{17}$ In all four psychopathology domains, suicide ideators showed greater symptoms, regardless of gender or pubertal status, controlling for age and socioeconomic status (Supplementary Material Figure S1). Among the psychopathology domains, the augmenting effect of suicidal ideation on symptom scores was more pronounced for mood-anxiety symptoms compared with the other domains (MMRM analysis treating domain as a within-subject variable, $P<0.001$ for all suicidal ideation $\times$ domain interaction), controlling for age, gender and socioeconomic status. Suicide ideators with current thoughts of suicide/death/dying reported greater symptom severity in all domains (Supplementary Material Figure S2).

\section{Association of level of function and overall cognitive performance with suicidal ideation}

Suicide ideators showed poorer functioning compared with nonsuicidal youth (mean C-GAS score \pm s.d. $68.6 \pm 13.7$ v. $80.4 \pm 11.3$, respectively, Cohen's $d=0.94$ ), controlling for lifetime depression or general psychopathology and for demographic covariates (Table 2). The association between suicidal ideation and poorer function was more pronounced in females (suicidal ideation $\times$ gender interaction, $P<0.001$ in all models) and more pronounced in pre-pubertal adolescence (suicidal ideation $\times$ puberty interaction, $P<0.016$ in model controlling for depression and demographics, trend-level $P$-values in other models). Suicide ideators with current thoughts of suicide/death/dying had lower level of functioning compared with suicide ideators without current thoughts (Supplementary Material Figure S3). Suicidal ideation was also associated with impaired function on the SOPS occupational function scale (odds ratio $4.118,95 \%$ CI $3.423-4.953, P<0.001$ ), an effect that remained significant after controlling for psychopathology (odds ratio 2.090, 95\% CI 1.708-2.558, $P<0.001$ ), and was more prominent in prepubertal suicide ideators compared with post-pubertal suicide

Table 1 Demographics and clinical characterisation of suicide ideators compared with non-suicidal youth

\begin{tabular}{|c|c|c|c|c|c|c|c|}
\hline & \multicolumn{2}{|c|}{$\begin{array}{c}\text { Total }^{\mathrm{a}} \\
(N=6151)\end{array}$} & \multicolumn{2}{|c|}{$\begin{array}{l}\text { Non-suicidal youth } \\
\qquad(N=5479)\end{array}$} & \multicolumn{2}{|c|}{$\begin{array}{l}\text { Suicide ideators }^{b} \\
\qquad(N=672)\end{array}$} & \multirow[b]{2}{*}{$P$} \\
\hline & Mean & s.d. & Mean & s.d. & Mean & s.d. & \\
\hline Age (years) & 15.8 & 2.7 & 15.6 & 2.7 & 16.8 & 2.6 & $<0.001^{\mathrm{c}}$ \\
\hline Socioeconomic status $^{d}$ & 0.02 & 1.00 & 0.03 & 0.99 & -0.05 & 1.02 & $0.032^{c}$ \\
\hline \multirow[t]{2}{*}{ General psychopathology ${ }^{\mathrm{d}}$} & 0.12 & 0.96 & 0.00 & 0.92 & 1.04 & 0.75 & $<0.001^{\mathrm{c}}$ \\
\hline & $N$ & $\%$ & $N$ & $\%$ & $N$ & $\%$ & \\
\hline Females & 3376 & 54.9 & 2694 & 54.1 & 411 & 61.2 & $0.001^{e}$ \\
\hline White & 3486 & 56.7 & 3121 & 57 & 365 & 54.3 & $0.191^{e}$ \\
\hline African American & 1992 & 32.4 & 1759 & 32.1 & 234 & 34.8 & $0.153^{\mathrm{e}}$ \\
\hline Post-pubertal & 2918 & 47.9 & 2515 & 45.9 & 432 & 64.3 & $<0.001^{\mathrm{e}}$ \\
\hline Depression $^{f}$ & 817 & 13.3 & 455 & 8.3 & 362 & 53.9 & $<0.001^{\mathrm{e}}$ \\
\hline
\end{tabular}




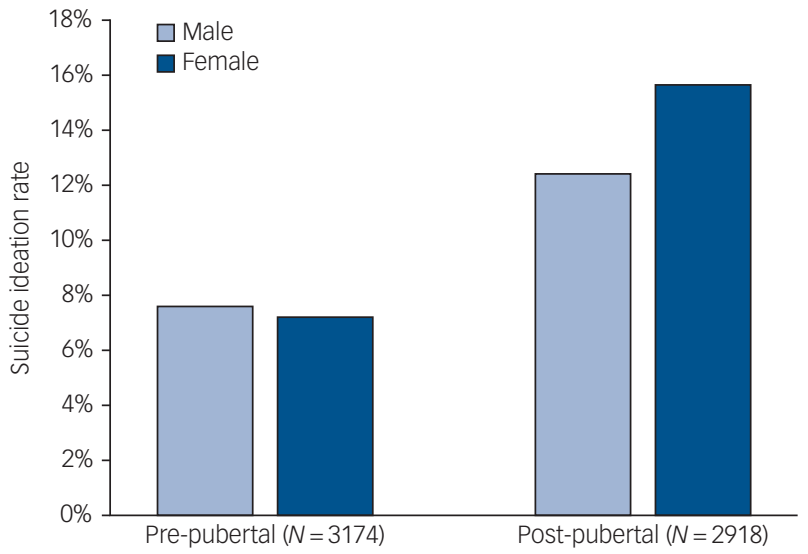

Fig. 1 Suicide ideation rates by gender and pubertal status.
$P=0.055$ or $P=0.051$, respectively). Separate models examining age effects without inclusion of pubertal status revealed similar main effects for suicidal ideation association with poorer function and better neurocognition, but no suicidal ideation $\times$ age interaction (Supplementary Material Table 1).

Sensitivity analysis that included suicide ideators $(N=672)$ with participants who endorsed having had a lot of thoughts about death/dying but did not endorse suicidal ideation $(N=795)$ revealed similar association of thoughts about suicide/death/dying with poorer level of function and better cognitive performance compared with non-suicidal youth. All main effects for thoughts about suicide/death/dying in association with function and cognition are the same in all models as the main analyses (Supplementary Material Table 2).

\section{Association of suicidal ideation with specific neurocognitive domains}

To probe for performance in specific neurocognitive domains across both males and females and spanning pre- and postpuberty, we compared the accuracy of suicide ideators versus controls in all cognitive tasks in an exploratory analysis (Fig. 2). We found that post-pubertal male suicide ideators outperformed their non-suicidal counterparts in tasks across cognitive domains including executive function (CPT and NBACK), episodic memory (verbal), complex reasoning (language and PMAT) and the social cognition-related age differentiation task (all FDR-corrected $P$-values $<0.05)$. In post-pubertal females, suicide ideators outperformed non-suicidal counterparts in the PMAT task (FDRcorrected $P<0.05)$. In pre-pubertal youth, no significant differences were observed in either males or females between suicide ideators compared with non-suicidal counterparts (Fig. 2). No differences were observed in the performance speed in post-pubertal youth in males or females (all FDR-corrected $P$-values $>0.12$, data not shown). Suicide ideators did not differ compared with non-suicidal youth in the number of false positive responses in the CPT, applied as a proxy marker for impulsivity (Supplementary Material Figure S4).

\begin{tabular}{|c|c|c|c|c|c|c|c|c|c|c|c|c|}
\hline & \multicolumn{3}{|c|}{ Model $A^{a}$} & \multicolumn{3}{|c|}{ Model $B^{b}$} & \multicolumn{3}{|c|}{ Model $C^{c}$} & \multicolumn{3}{|c|}{ Model $\mathrm{D}^{\mathrm{d}}$} \\
\hline & $\beta$ & $t$ & $P$ & $\beta$ & $t$ & $P$ & $\beta$ & $t$ & $P$ & $\beta$ & $t$ & $P$ \\
\hline \multicolumn{13}{|l|}{ Level of function (C-GAS) } \\
\hline suicide ideation & -0.306 & -25.120 & $<0.001^{*}$ & -0.307 & -25.157 & $<0.001^{*}$ & -0.232 & -17.652 & $<0.001^{*}$ & -0.182 & -15.093 & $<0.001 *$ \\
\hline Gender effect & 0.027 & 2.193 & $0.028^{*}$ & 0.026 & 2.118 & $0.034^{*}$ & 0.042 & 3.412 & $0.001 *$ & 0.022 & 1.866 & 0.062 \\
\hline Puberty effect & 0.003 & 0.205 & 0.838 & 0.002 & 0.191 & 0.848 & 0.016 & 1.302 & 0.193 & 0.003 & 0.255 & 0.799 \\
\hline Suicidal ideation $\times$ gender & & 3.091 & $0.002^{*}$ & & 3.134 & $0.002^{*}$ & & 2.378 & $0.017^{*}$ & & 3.494 & $<0.001 *$ \\
\hline Suicidal ideation $\times$ puberty & & 1.816 & 0.069 & & 1.808 & 0.071 & & 2.400 & $0.016^{*}$ & & 1.697 & 0.090 \\
\hline Gender $\times$ puberty & & 0.937 & 0.349 & & 1.057 & 0.291 & & 1.376 & 0.169 & & 0.999 & 0.318 \\
\hline Suicidal ideation $\times$ gender $\times$ puberty & & 0.623 & 0.533 & & 0.578 & 0.563 & & 0.574 & 0.566 & & 0.352 & 0.725 \\
\hline \multicolumn{13}{|l|}{$\begin{array}{l}\text { Cognitive performance (overall accuracy } \\
\text { on CNB) }\end{array}$} \\
\hline Suicide ideation & 0.037 & 3.086 & $0.002^{*}$ & 0.035 & 2.883 & $0.004^{*}$ & 0.027 & 2.055 & $0.040^{*}$ & 0.06419 & 5.031 & $0.000 *$ \\
\hline Gender effect & 0.006 & 0.457 & 0.648 & 0.007 & 0.549 & 0.583 & 0.008 & 0.614 & 0.539 & 0.007 & 0.562 & 0.574 \\
\hline Puberty effect & 0.038 & 3.057 & $0.002^{*}$ & 0.039 & 3.135 & $0.002^{*}$ & 0.035 & 2.837 & $0.005^{*}$ & 0.03895 & 3.166 & $0.002 *$ \\
\hline Suicidal ideation $\times$ gender & & 1.072 & 0.284 & & 1.206 & 0.228 & & 1.166 & 0.243 & & 1.101 & 0.271 \\
\hline Suicidal ideation $\times$ puberty & & 1.994 & $0.046^{*}$ & & 2.021 & 0.043 & & 1.920 & 0.055 & & 1.952 & 0.051 \\
\hline Gender $\times$ puberty & & 0.296 & 0.767 & & 0.616 & 0.538 & & 0.241 & 0.810 & & 0.296 & 0.767 \\
\hline Suicidal ideation $\times$ gender $\times$ puberty & & 0.550 & 0.582 & & 0.708 & 0.479 & & 0.541 & 0.589 & & 0.623 & 0.534 \\
\hline \multicolumn{13}{|c|}{$\begin{array}{l}\text { Linear regression models with level of function (C-GAS score, top) or overall cognitive performance (CNB accuracy score, bottom) as the dependent variable and suicidal ideation, gender, } \\
\text { puberty and their interactions as the independent variables, controlling for age (puberty regressed), socioeconomic status, race, lifetime depression and general psychopathology factor } \\
\text { score. C-GAS, Children's Global Assessment Scale; CNB, Penn Computerized Neurocognitive Battery. } \\
\text { a. Model A controls for age and socioeconomic status. } \\
\text { b. Model B controls for age, race and parents' separation status. } \\
\text { c. Model C controls for age, socioeconomic status and lifetime depression. } \\
\text { d. Model D controls for age, socioeconomic status and general psychopathology. } \\
\text { * } P<0.05 \text {. }\end{array}$} \\
\hline
\end{tabular}



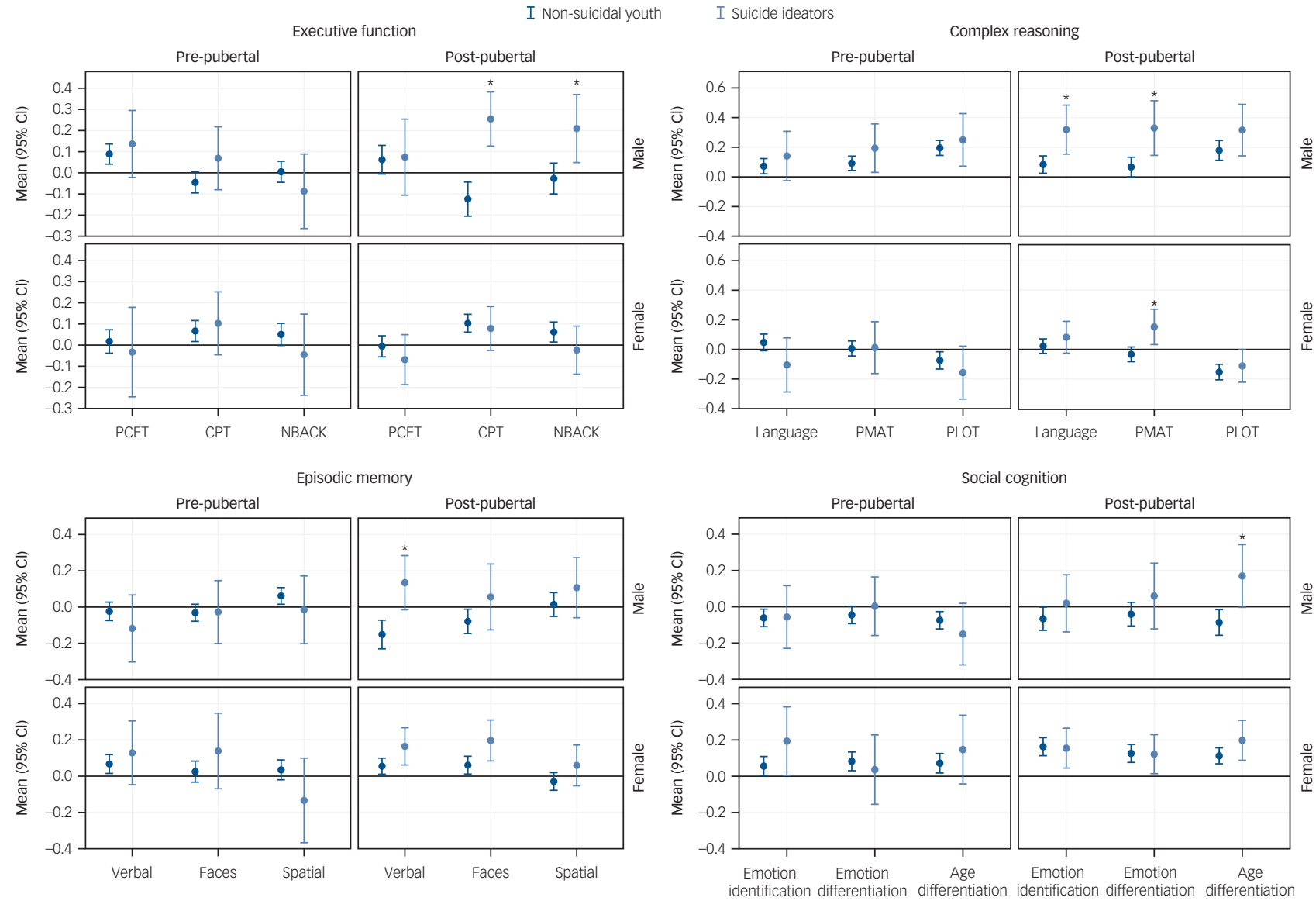

Fig. 2 Exploratory analysis of performance on cognitive tasks in suicide ideators versus controls. CPT, Continuous Performance Test; NBACK, Letter N-Back; PCET, Penn Conditional Exclusion Test; PLOT, Penn Line Orientation Test; PMAT, Penn Matrix Reasoning Test.

\section{Discussion}

We describe a dissociation between function and neurocognitive performance in youth with suicidal ideation compared with their non-suicidal counterparts. Suicide ideators show poorer functioning, documented by two clinical scales, while demonstrating higher cognitive accuracy, when controlling for lifetime depression or general psychopathology. Specifically, post-pubertal male suicide ideators show better cognitive performance compared with their non-suicidal counterparts, with small to medium effect sizes. Better performance was evident in multiple cognitive tasks across several domains including executive function, episodic memory, complex reasoning and social cognition. Given the literature suggesting association of suicidal ideation with lower cognitive function in young adulthood, ${ }^{10,25,26}$ the higher cognitive performance in the suicide ideators in the current study is surprising. However, our findings are consistent with a European study describing a positive association between IQ in boys at age 8 with suicidal ideation in late adolescence, ${ }^{11}$ and with report of positive association of IQ at age 7 with suicidal ideation in later life (age 17-49), described in people within an average IQ range. ${ }^{27}$ In addition, in adults with depression, those with a history of suicidal behaviour perform better on some executive functioning tasks. ${ }^{28}$

The overall rate of reported suicidal ideation in our sample was $10.9 \%$, consistent with the National Comorbidity Survey Replication Adolescent Supplement study describing 12.1\% suicidal ideation in an American community sample aged $13-18 .^{5}$ Importantly, to avoid misclassification and to enhance generalisability of our findings, we excluded from the main analysis participants who endorsed having frequent thoughts about death or dying, but denied suicidal thoughts, which may account for the relatively low suicidal ideation rate in our sample compared with higher rates reported elsewhere. ${ }^{29}$ The current study population enabled us to investigate gender-specific and puberty associations with suicidal ideation. In line with previous studies, we found no gender differences before puberty and a higher rate of suicidal ideation in females post-puberty. ${ }^{29}$ The higher levels of psychopathology we found in the suicide ideators was most prominent for mood and anxiety symptoms, as has been previously reported in youth. ${ }^{30}$ The consistency of our findings with reported rate of suicidal ideation, gender and puberty effects and associated psychopathology with youth suicidal ideation provide support for the generalisability of our findings.

Suicidal ideation was associated with lower functioning in two scales, the C-GAS (large effect size, $d=0.94$ ) and the SOPS occupational function scale (moderate to large effect size, odds ratio 4.12). As the study participants were largely school aged, these findings are comparable to the reported association of poorer academic attainment with later life suicidal behaviours. ${ }^{31-35}$ Our findings of suicidal ideation $\times$ puberty interaction in association with poorer function when controlling for lifetime depression suggest that, specifically for younger individuals, suicidal ideation is associated with even more detrimental effects on functioning; suggesting that this population may be more vulnerable to the burden of suicidal ideation. This finding may have mental health policy implications as we show that, in a community non-help-seeking sample, pre-pubertal youth with suicidal ideation may already be demonstrating a detrimental developmental trajectory and may represent a population 
that should be identified through early (pre-pubertal) screening and be offered treatment to prevent further functional deterioration.

In contrast to the negative association of suicidal ideation with level of function, there was an opposite trend of association of suicidal ideation with cognitive function, as suicide ideators showed better cognitive performance. This effect was most prominent postpuberty, especially in males (small to medium effect size, $d=0.32$ ). Importantly, associations of suicidal ideation with poorer function and better cognitive performance remained significant after controlling for key confounders like history of depression or general psychopathology (recently reported to contribute to long-term outcomes in youth $^{36}$ ) and for current thoughts of suicide/death/dying, suggesting that there may be a specific link between better cognitive performance and suicidal ideation in community youth.

Although our findings of suicidal ideation association with poorer function level were expected, the association of suicidal ideation with better neurocognitive performance highlights the complex association between suicidal ideation and neurocognitive development in adolescence. Notably, lower neurocognitive performance in young adults was previously suggested as a diathesis to subsequent suicide-related outcomes. ${ }^{25,26}$ Our findings may point to specific associations of suicidal ideation with better neurocognition in a specific time window during adolescence that was not captured in studies of young adults. In addition, studies have shown that lower IQ in early adolescence was predictive of suicide attempts ${ }^{35}$ and with suicide in adulthood in males. ${ }^{31}$ In our study, we examined associations of neurocognition with suicidal ideation, as we did not have data of suicide attempts or longitudinal data on suicide outcomes. It is possible that suicide-related phenotypes are differentially associated with neurocognitive performance during adolescence (i.e. youths with suicidal ideation have better neurocognition whereas youth with suicide attempts have poorer neurocognition). Longitudinal studies examining trajectories of neurocognition and suicide-related phenotypes from early adolescence to adulthood are needed to determine the causal pathways. Nonetheless, our findings that in mid-adolescence, suicidal ideation is associated with better, rather than poorer, neurocognitive performance may have clinical implication as it may suggest that high intelligence should not be viewed by clinicians as a protective factor from subsequent suicide risk.

\section{Limitations}

First, the cross-sectional design prevents making causal inferences, although including pubertal status in the analyses adds a developmental perspective. Second, the clinical evaluation of suiciderelated phenotypes was limited and included two questions (thought about killing oneself and thought a lot about death/ dying), without detailed probes for additional data such as history of suicide attempts. To address this limitation and avoid misclassification, we included in the main analysis suicidal ideation youth who directly endorsed having ever had thoughts about killing themselves (excluding participants that endorsed having though a lot about death/dying but did not endorse thoughts about killing themselves), in line with a recent report suggesting high sensitivity and specificity of using a single item regarding suicidal ideation compared with more elaborate assessment of suicide-related measures. ${ }^{37}$ Third, our clinical evaluation included assessment of lifetime history of depressive episodes and not current depressive phenotyping, thus it was not possible to control for current depression in the models predicting cognition and function. Fourth, the C-GAS function level rating may be somewhat confounded with suicidal ideation in the past year and thus overestimate the negative effect size associated with suicidal ideation. Nonetheless, we show a compelling association of suicidal ideation with impaired function by using the SOPS occupational function scale, which is independent of suicide-related measures and, in children, taps into school function. Fifth, as the PNC included participants with cognitive capacity to complete the neurocognitive battery, our findings do not address suicidal ideation and neurocognition associations in youth with intellectual disability. Finally, we acknowledge that the PNC effort was not designed specifically to study phenotypes associated with youth suicide ideation. Nonetheless, we show that the PNC presents an unprecedented opportunity to unravel neurocognitive correlates of suicide ideation in a large, community sample of youth not seeking mental-health help.

Ran Barzilay, MD, PhD (ID), Research Scientist, Lifespan Brain Institute, Children's Hospital of Philadelphia and Penn Medicine; and Department of Child and Adolescent Psychiatry and Behavioral Sciences, Children's Hospital of Philadelphia, USA; Monica E. Calkins, PhD, Associate Professor, Neuropsychiatry Section, Department of Psychiatry, Perelman School of Medicine, University of Pennsylvania, USA; Tyler M. Moore, PhD, Research Associate, Neuropsychiatry Section, Department of Psychiatry, Perelman School of Medicine, University of Pennsylvania, USA; Rhonda C. Boyd, PhD, Associate Professor, Department of Child and Adolescent Psychiatry and Behavioral Sciences, Children's Hospital of Philadelphia, USA; Jason D. Jones, PhD, Research Scientist, Department of Child and Adolescent Psychiatry and Behavioral Sciences, Children's Hospital of Philadelphia, USA; Tami D. Benton, MD, Associate Professor, Chair of the Department of Child and Adolescent Psychiatry and Behavioral Sciences, Children's Hospital of Philadelphia, USA; Maria A. Oquendo, MD, PhD, Professor, Chair of the Department of Psychiatry, Perelman School of Medicine, University of Pennsylvania, USA; Ruben C. Gur, PhD Professor, Lifespan Brain Institute, Children's Hospital of Philadelphia and Penn Medicine; and Director of Neuropsychiatry Section, Department of Psychiatry, Perelman School of Medicine, University of Pennsylvania, USA: Raquel E. Gur MD, PhD, Professor, Director of Lifespan Brain Institute, Children's Hospital of Philadelphia and Penn Medicine; and Neuropsychiatry Section, Department of Psychiatry, Perelman School of Medicine, University of Pennsylvania, USA

Correspondence: Ran Barzilay, 10th floor, Gates Building, Hospital of the University of Pennsylvania, 34th and Spruce Street, Philadelphia, PA 19104, USA. Email: barzilayr@email.chop.edu

First received 25 Oct 2018, final revision 23 Jan 2019, accepted 2 Feb 2019

\section{Supplementary material}

Supplementary material is available online at https://doi.org/10.1192/bjp.2019.55.

\section{Funding}

This work was supported by National Institutes of Health grants MH-107235, MH-089983, MH096891 and MH-P50MH06891; the Dowshen Neuroscience fund; and the Lifespan Brain Institute of Children's Hospital of Philadelphia and Penn Medicine, University of Pennsylvania. The funding organisations had no role in the design and conduct of the study; collection, management, analysis and interpretation of the data; preparation, review or approval of the manuscript; and decision to submit the manuscript for publication.

\section{Acknowledgements}

The authors thank Kathleen Merikangas, PhD, and Marcy Burstein, PhD, Genetic Epidemiology Research Branch, Intramural Research Program, National Institute of Mental Health for assistance in development of GOASSESS: they received no compensation from the funding sponsor. We thank Kosha Ruparel, the data lead of the Brain and Behavior Laboratory at the Neuropsychiatry Section in the Department of Psychiatry at Perelman School of Medicine, University of Pennsylvania, whose individual contribution to data management made this work possible. We thank participants and families; staff of the Brain Behavior Laboratory, University of Pennsylvania, Philadelphia, for their contribution to data generation; study assessors and recruiters for their invaluable contributions to data collection; and staff from the Center of Applied Genomics, Children's Hospital of Philadelphia, Philadelphia, Pennsylvania, for the genomics interphase with brain behaviour measures.

\section{References}

1 Hawton $\mathrm{K}$, Saunders KE, O'Connor RC. Self-harm and suicide in adolescents. Lancet 2012; 379: 2373-82.

2 World Health Organisation (WHO). WHO Suicide Data. WHO, 2016 (http://www. who.int/mental_health/prevention/suicide/suicideprevent/en/).

3 Sveticic J, De Leo D. The hypothesis of a continuum in suicidality: a discussion on its validity and practical implications. Ment IIIn 2012; 4: e15. 
4 Adrian M, Miller AB, McCauley E, Vander Stoep A. Suicidal ideation in early to middle adolescence: sex-specific trajectories and predictors. J Child Psychol Psychiatry 2016; 57: 645-53

5 Nock MK, Green JG, Hwang I, McLaughlin KA, Sampson NA, Zaslavsky AM, et al. Prevalence, correlates, and treatment of lifetime suicidal behavior among adolescents. JAMA Psychiatry 2013; 70: 300-10.

6 Herba CM, Ferdinand RF, Van der Ende J, Verhulst FC. Long-term association of childhood suicide ideation. J Am Acad Child Adolesc Psychiatry 2007; 46 1473-81.

7 Copeland WE, Goldston DB, Costello EJ. Adult associations of childhood suicidal thoughts and behaviors: a prospective, longitudinal analysis. J Am Acad Child Adolesc Psychiatry 2017; 56: 958-65.e4.

8 Fergusson DM, Horwood L, Ridder EM, Beautrais AL. Suicidal behaviour in adolescence and subsequent mental health outcomes in young adulthood. Psychol Med 2005; 35: 983-93.

9 Reinherz HZ, Tanner JL, Berger SR, Beardslee WR, Fitzmaurice GM. Adolescent suicidal ideation as predictive of psychopathology, suicidal behavior, and compromised functioning at age 30. Am J Psychiatry 2006; 163: 1226-32.

10 Alati R, Gunnell D, Najman J, Williams G, Lawlor D. Is IQ in childhood associated with suicidal thoughts and attempts? Findings from the Mater University Study of Pregnancy and its outcomes. Suicide Life Threat Behav 2009; 39: 282-93.

11 Chang S-S, Chen Y-Y, Heron J, Kidger J, Lewis G, Gunnell D. IQ and adolescent self-harm behaviours in the ALSPAC birth cohort. J Affect Disord 2014; 152154: $175-82$

12 Glaser B, Gunnell D, Timpson NJ, Joinson C, Zammit S, Smith GD, et al. Age- and puberty-dependent association between IQ score in early childhood and depressive symptoms in adolescence. Psychol Med 2011; 41: 333-43.

13 Calkins ME, Merikangas KR, Moore TM, Burstein M, Behr MA, Satterthwaite TD, et al. The Philadelphia Neurodevelopmental Cohort: constructing a deep phenotyping collaborative. J Child Psychol Psychiatry 2015; 56: 1356-69.

14 Moore TM, Martin IK, Gur OM, Jackson CT, Scott JC, Calkins ME, et al. Characterizing social environment's association with neurocognition using census and crime data linked to the Philadelphia Neurodevelopmental Cohort. Psychol Med 2016; 46: 599-610.

15 Jones JD, Boyd RC, Calkins ME, Barzilay R, Ahmed A, Moore TM, et al. Parentadolescent agreement about adolescents' suicidal thoughts. Pediatrics 2019; 143: e20183071.

16 Kaufman J, Birmaher B, Brent D, Rao U, Flynn C, Moreci P, et al. Schedule for affective disorders and schizophrenia for school-age children-present and lifetime version (K-SADS-PL): initial reliability and validity data. J Am Acad Child Adolesc Psychiatry 1997; 36: 980-8.

17 Shanmugan S, Wolf DH, Calkins ME, Moore TM, Ruparel K, Hopson RD, et al. Common and dissociable mechanisms of executive system dysfunction across psychiatric disorders in youth. Am J Psychiatry 2016; 173: 517-26.

18 Shaffer D, Gould MS, Brasic J, Ambrosini P, Fisher P, Bird H, et al. A Children's Global Assessment Scale (CGAS). 1983; 40: 1228-31.

19 Miller TJ, McGlashan TH, Woods SW, Stein K, Driesen N, Corcoran CM, et al. Symptom assessment in schizophrenic prodromal states. Psychiatr Q 1999; 70: $273-87$.

20 Satterthwaite TD, Shinohara RT, Wolf DH, Hopson RD, Elliott MA, Vandekar SN et al. Impact of puberty on the evolution of cerebral perfusion during adolescence. Proc Natl Acad Sci USA 2014; 111: 8643-8.

21 Gur RC, Richard J, Calkins ME, Chiavacci R, Hansen JA, Bilker WB, et al. Age group and sex differences in performance on a computerized neurocognitive battery in children age 8-21. Neuropsychology 2012; 26: 251-65.
22 Wilkinson G. WRAT 4: Wide Range Achievement Test Professional Manual (4th edn). Psychological Assessment Resources Inc., 2006 (http://www.worldcat. org/title/wrat-4-wide-range-achievement-test-professional-manual/oclc/ 165083815)

23 Epstein JN, Erkanli A, Conners CK, Klaric J, Costello JE, Angold A. Relations between continuous performance test performance measures and ADHD behaviors. J Abnorm Child Psychol 2003; 31: 543-54.

24 Pinheiro J, Bates D, DebRoy S, Sarkar D and R Core Team. nlme: Linear and Nonlinear Mixed Effects Models. R package version 3.1-137, 2018 (https:// CRAN.R-project.org/package=nlme)

25 Gunnell D, Magnusson PKE, Rasmussen F. Low intelligence test scores in 18 year old men and risk of suicide: cohort study. BMJ 2005; 330: 167

26 Naifeh JA, Nock MK, Ursano RJ, Vegella PL, Aliaga PA, Fullerton CS, et al. Neurocognitive function and suicide in U.S. army soldiers. Suicide Life Threat Behav 2017; 47: 589-602.

27 Hung GC-L, Pietras SA, Carliner H, Martin L, Seidman L, Buka SL, et al. Cognitive ability in childhood and the chronicity and suicidality of depression. Br J Psychiatry 2016; 208: 120-7.

28 Keilp JG, Gorlyn M, Russell M, Oquendo MA, Burke AK, Harkavy-Friedman J, et al. Neuropsychological function and suicidal behavior: attention control, memory and executive dysfunction in suicide attempt. Psychol Med 2013; 43: 539-51.

29 Cha CB, Franz PJ, Guzmán EM, Glenn CR, Kleiman EM, Nock MK. Annual Research Review: suicide among youth - epidemiology, (potential) etiology, and treatment. J Child Psychol Psychiatry 2018; 59: 460-82.

30 Gould MS, King R, Greenwald S, Fisher P, Schwab-Stone M, Kramer R, et al. Psychopathology associated with suicidal ideation and attempts among children and adolescents. J Am Acad Child Adolesc Psychiatry 1998; 37 915-23.

31 Andersson L, Allebeck P, Gustafsson J-E, Gunnell D. Association of IQ score and school achievement with suicide in a 40-year follow-up of a Swedish cohort. Acta Psychiatr Scand 2008; 118: 99-105.

32 Gunnell D, Löfving S, Gustafsson J-E, Allebeck P. School performance and risk of suicide in early adulthood: follow-up of two national cohorts of Swedish schoolchildren. J Affect Disord 2011; 131: 104-12.

33 Kosidou K, Dalman C, Fredlund P, Lee BK, Galanti R, Isacsson G, et al. School performance and the risk of suicide attempts in young adults: a longitudinal population-based study. Psychol Med 2014; 44: 1235-43.

34 Kosidou K, Dalman C, Fredlund P, Magnusson C. School performance and the risk of suicidal thoughts in young adults: population-based study. PLOS One 2014; 9: e109958.

35 Sörberg Wallin A, Zeebari Z, Lager A, Gunnell D, Allebeck P, Falkstedt D. Suicide attempt predicted by academic performance and childhood IQ: a cohort study of 26000 children. Acta Psychiatr Scand 2018; 137: 277-86.

36 Pettersson $\mathrm{E}$, Lahey $\mathrm{BB}$, Larsson $\mathrm{H}$, Lichtenstein $\mathrm{P}$. Criterion validity and utility of the general factor of psychopathology in childhood: predictive associations with independently measured severe adverse mental health outcomes in adolescence. J Am Acad Child Adolesc Psychiatry 2018; 57: $372-83$.

37 Millner AJ, Lee MD, Nock MK. Single-item measurement of suicidal behaviors: validity and consequences of misclassification. PLOS One 2015; 10: e0141606 